This is the accepted version of the manuscript published by Springer in Current Psychology, available at https://doi.org/10.1007/s12144-019-00507-3

Citation:

Aldrovandi, S., Kusev, P., Hill, T., \& Vlaev, I. (in press). From Gloom to Doom: Financial Loss and Negative Affect Prime Risk Averse Preferences. Current Psychology. DOI: 10.1007/s12144019-00507-3

\title{
From Gloom to Doom: Financial Loss and Negative Affect Prime Risk Averse Preferences
}

\author{
Silvio Aldrovandi ${ }^{a^{*}}$, Petko Kusev ${ }^{\mathrm{b}}$, Tetiana Hill ${ }^{\mathrm{c}}$, and Ivo Vlaev ${ }^{\mathrm{d}}$ \\ ${ }^{a}$ Department of Psychology, Birmingham City University, Birmingham, United Kingdom; \\ ${ }^{b}$ Department of Management, University of Huddersfield, Huddersfield, United Kingdom; \\ ${ }^{c}$ Hertfordshire Business School, University of Hertfordshire, Hatfield, United Kingdom; \\ ${ }^{d}$ Department of Surgery and Cancer, Imperial College, London, United Kingdom.
}

*Corresponding author: Silvio Aldrovandi, Department of Psychology, Birmingham City University, 4 Cardigan Street, Birmingham, B4 7BD, United Kingdom. Telephone: +44(0)121

3316547. Email: Silvio.Aldrovandi@bcu.ac.uk. Orcid: 0000-0002-5604-8551 


\begin{abstract}
Previous research has shown that risk preferences are sensitive to the financial domain in which they are framed. In the present study we explore whether the effect of valence priming on risk taking is moderated by the financial context under consideration. A total of 260 participants completed an online questionnaire where risky choices were elicited for seven different financial scenarios. Participants were allocated to different valence (neutral, positive or negative) and arousal (low or high) priming conditions. Two factors were extracted: Factor 1 (Negative) included insurance and possibility of loss, whilst Factor 2 (Positive) included the remaining five scenarios (investment, salary, pension, possibility of gain, and mortgage). Moreover, only negative priming — regardless of arousal level—influenced people's risky choices by inducing more risk-averse behavior; this effect was confined only to loss and insurance domains. The findings call into question the generalizability of priming effects on different financial context and show that the effects of priming on financial risk taking are sensitive to the financial context under consideration.
\end{abstract}

Keywords: Financial risk; context effects; priming; risk preferences 


\section{Introduction}

A consistent claim from behavioral decision researchers is that, contrary to the assumptions of classical economics, preferences are not stable and inherent in individuals but are constructed "on the fly" and are strongly influenced by context and the available choice options (Kusev et al. 2017, 2019; Slovic 1995; Vlaev et al. 2010, 2011). Moreover, risky decisionmaking has been shown to be determined by available information (e.g., the specified probability) and by experience (e.g., pre-experimental beliefs about event frequencies; Kusev et al. 2009; van Schaik, Kusev, and Juliusson 2011). As a result, the construction of risk preferences is influenced by the accessibility of events in memory (e.g., Aldrovandi et al. 2015; Kusev, van Schaik, and Aldrovandi 2012; Kusev and van Schaik 2011).

In addition to contextual information and memory retrieval, a strand of research suggests that situational affect exerts an independent influence on preferences, thus further hampering their stability (e.g., Rottenstreich and Hsee 2001). Zajonc (1980) showed that the mere exposure to stimuli increased their familiarity and consequently their attractiveness and famously claimed that "preferences need no inferences". Zajonc's work suggests that affective judgments may be fairly independent of the sort of perceptual and cognitive operations commonly assumed to be the basis of people's preferences (see also Finucane et al. 2000; Loewenstein et al. 2001). Consequently, the reasons people offer for their choices may not be those that drove their decision-making (see also Erb, Bioy, and Hilton 2002; Johansson et al. 2005).

Research on the role of affect on risk taking has offered contrasting findings. In priming studies, it has been shown that negative affect associated with a target stimulus can increase its perceived risk and lower its perceived benefits, while the opposite is true for positive priming (e.g., Finucane et al. 2000). Indeed, some findings show that positive mood or state is associated with more risk taking - and that, if in a positive state, participants tend to report higher subjective probabilities for positive events and lower subjective probabilities for negative events. Mood congruency (e.g., Meyer et al. 1992) has been invoked as a possible explanation for these effects: People seem more likely to retrieve mood-congruent memories than mood incongruent information, which in turns influences their choices as memory drives decision making (e.g., Kusev et al. 2009). However, Isen and her colleagues (e.g., Isen, Nygren, and Ashby 1988; Kahn and Isen 1993) showed that people in a positive affective state can actually be more riskaverse - especially in the presence of high stakes — while they are more risk-seeking if in a 
negative affective state (see also Mano 1994). Relatedly, other studies have suggested that positive mood can reduce risk seeking (Isen et al. 1988; Arkes, Herren, and Isen 1988). Amongst the explanations that have been proposed to account for these effects, the mood maintenance effect relates to the tendency to maintain current positive feeling by avoiding choices that could compromise them (e.g., Isen 2000), which is associated to a lower willingness to take risks. Similarly, the mood repair effect ascribes the greater risk taking associated with negative affective state to people actively seeking decisions that have the potential to change such state (e.g., Forgas 1991).

More recently, investigations have sharpened their focus on the interplay between contextual factors and affect in risky decision-making. For example, Seo, Goldfarb, and Barrett (2010) analyzed the influence of affective state on framing effects, people's tendency to make risk-averse choices for gains and risk-seeking choices for losses (e.g., Tversky and Kahneman 1979). People's choices in an investment simulation revealed an attenuating influence of affective state on framing effects in line with the mood congruency hypothesis. More specifically, risk aversion was diminished for gains when people experienced positive feelings, whilst risk seeking behavior for loss-framed prospects decreased when people experienced negative feelings. These findings suggest that affect shapes the way in which contextual factors (e.g., framing effects) influence risk preferences.

\subsection{Current Study}

In order to further explore how context and affect interact in influencing risk preferences, here we explore context as the financial domain in which a specific prospect is framed and affect as the result of valence priming. Indeed, context effects often prompt complex mental statesincluding both cognitions and affect - in risk taking, which results in choice patterns that violate normative assumptions about stable and generalizable risk attitudes. Previous research has shown that context - as represented by different financial products — does indeed hinder the stability of risk preferences. Vlaev et al. (2010) conducted an analysis of context effects in financial domains and investigated whether people are either sensitive to the scenario at hand or whether they display the same risk attitudes regardless of the financial scenario. The results supported the former as participants' risk preferences were sensitive to the financial scenario under consideration, whose valence influenced participants' decision-making. Risk preferences were 
stable within positive financial domains (e.g., salary) and within negative ones (e.g., gamble to lose), but not particularly so across them.

Thus, a question that is yet unanswered concerns whether affect and contextual valence interact or not in financial risk taking. Does priming influence risk taking regardless of the financial scenario at hand-or are priming effects moderated by the contextual valence of the financial product under consideration? If a person is negatively primed, would she be willing to take fewer risky decisions for, say, both a relatively 'positive' domain such as salary and a 'negative' product (e.g., insurance, where the focus is on potential losses)—or just for either?

There are at least two reasons as to why answering the above question may contribute to the literature on financial risk taking. First, if priming effects are moderated by the financial context, sustaining the stability of risk preferences becomes even more arduous. Second, most previous studies did not systematically investigate the influence of affect on decision-making for different financial products. For example, some studies limited their investigation to varied activities characterized by a potential for risk (e.g., betting on horses and buying cars; Erb et al., 2002), to single financial contexts (e.g., gambles to win money, Moore and Chater 2003; investment, Gilad and Kliger 2008), or to hypothetical monetary scenarios (e.g., Kahn and Isen 1993).

Based on the previously discussed literature, a series of hypotheses can be put forward. According to the mood congruency hypothesis (e.g., Meyer et al. 1992), affective states influence the perceived probability of specific outcomes depending on their valence. Thus, positive moods may reduce risk aversion for positively framed financial products (e.g., salary) by increasing the subjective probability of the potential gain. Similarly, mood congruency would increase the subjective probability of the potential loss in a negatively framed product (e.g., insurance), thus reducing risk seeking. On the other hand, mood maintenance and mood repair effects (e.g., Forgas 1991) would predict the opposite pattern. In order to maintain the current positive state when faced with a positive financial scenario and due to a positive mood, people would display less risk seeking choices. For a choice framed as a negative financial product and faced whilst in a negative mood, an increase in risk seeking behavior would be predicted due to the underlying aim to change the current negative state. 


\section{Method}

We investigated participants' financial risk preferences on a series of choices where different payoffs and probabilities were varied across trials as in Vlaev et al. (2010). The influence of priming valence on financial risk preferences was investigated in relation to the valence of the financial context in which the choices were framed.

\subsection{Participants}

A total of 260 respondents (148 males) took part in the online study; participants' age ranged from 18 to $82(M=46.8, S D=15.9)$. Participants were granted Maximiles ${ }^{\circledR}$ points in exchange for their participation. A total of $44.6 \%$ of participants indicated secondary school as the highest level of education, whilst $23.1 \%$ held a bachelor degree and $10.0 \%$ a master degree. Participants indicated a very wide range of profession, ranging from unskilled to managerial positions; a total of 33 participants (i.e., $12.7 \%$ of the total) were retired. Participants were randomly assigned to valence and priming conditions; a relatively balanced design was achieved as a range from 42 to 50 participants were allocated to each of the six design cells.

\subsection{Design and Materials}

The study was a questionnaire-based survey that employed hypothetical measures of risk preferences, whereby respondents made choices in seven different financial scenarios as in Vlaev et al. (2010): Gamble to win, gamble to lose, mortgage, salary, investment, insurance, and pension (for full descriptions, see Appendix A). Despite the decision-making products were framed as hypothetical choices, we made sure that they were analogous real financial products on the market. It is also important to notice that the same risky questions were asked within each

of the seven different financial contexts; hence the description of the problem was the only factor that could have affected participants' risk preferences, together with the priming manipulation. Also, we controlled for absolute wealth by counterbalancing across participants the amounts for the financial scenarios.

The preparation of the choice stimuli followed exactly Vlaev et al. (2010). On each trial, participants were asked to make a choice between a sure outcome and a risky prospect; each pair of options was presented as two-pie charts (see examples in Appendix B). As in Vlaev et al. (2010), the risky option was operationalized by crossing four probabilities $(20 \%, 40 \%, 60 \%$, and $80 \%$ ) with four amounts ( $£ 100, £ 200, £ 300$, and $£ 400$ ) to create a total of 16 choices. The accompanying sure outcome was generated by using a power law utility function with power $\gamma$ 
(gamma), in a way that a person with power $\gamma$ would be indifferent between the sure outcome and the risk. As in Vlaev et al. (2010), we used the following equation:

$$
y=x p^{1 / \gamma},
$$

where $\gamma$ represents the sure amount and the risky prospect is " $p$ chance of $x$ ". Thus, $\gamma$ describes the curvature of a hypothetical power law utility function, $u(x)=x^{\gamma}$. For a neutral person, $\gamma=1$, whilst smaller values of $\gamma$ indicate greater risk aversion. Four levels of $\gamma(0.35$, $0.50,0.65$, and 0.80 ) were used in this experiment to generate different risky behaviors whereby, for example, for some questions even the most risk averse participant would choose the risky prospect. Also, the values of $\gamma$ we used were intended to allow for participants in the middle of the risk-aversion continuum to choose a mixture of sure amounts and risky prospects (see Vlaev et al. 2010 for further details).

Levels of $\gamma$ were randomly assigned to gambles with the constraint that each level of $\gamma$ occurred once for each amount and once for each probability. Also, we presented 16 gambles per financial domain and made sure that, at least, each scenario presented all four levels of $\gamma$ paired with every monetary amount and probability. We also used four different orders of the four $\gamma$ levels across the 16 gambles, by creating a priori seven defined presentation orders and ensuring that each financial domain was presented in the context of four different gamble sets, which controlled for possible interactions between each financial domain and a particular gamble set. As in Vlaev et al. (2010), we coded each risky choice as 1 and each choice for the sure outcome as 0 , with lower proportion of risky choices indicating greater risk aversion.

In order to manipulate priming, the gambles were presented with different backgrounds images, whereby each participant completed the whole survey with one picture in the background (for a similar priming procedure, see Vohs, Mead, and Goode 2006). Arousal (low or high) and valence (neutral, negative or positive) of the pictures were manipulated orthogonally between participants. Normative data about arousal and valence of pictures were taken from the Affective Picture System database (IAPS; Lang, Bradley, and Cuthbert 1999). More specifically, values were taken from the bottom quartile (for low arousal and negative valence), median (for the neutral valence), and top quartile (for high arousal and positive valence) within the database dimensions of valence and arousal; normative ratings ranged from 1 to 9 (see Appendix C). 
In order to assess participants' affective state, the 60 questions from the PaNAS (Watson, Clark, and Tellegen 1988) were answered both before and after the risky choice task by each participant. Finally, two additional measures were used in order to measure participants' (a) subjective evaluations of the background picture and (b) self-assessed expertise with each of the seven financial scenarios. For the former, after the completion of post-test PaNAS, participants were asked to rate what emotion/how they felt-(1) negative, unhappy, (2) positive, happy, (3) calm, unaroused and (4) excited, aroused — as a consequence of viewing the background picture. Ratings were collected along a Likert scale anchored at 1 ("very slightly or not at all") and 7 (“extremely"). We then asked respondents to rate how much experience they had with each of the encountered seven financial scenarios; participants self-assessed their expertise using a Likert scale ranging from 0 ("none") to 9 ("a lot").

\subsection{Procedure}

The study was conducted in accordance with the Declaration of Helsinki, and the protocol was approved by the Ethics Committee of City University London. As participants were redirected to the survey link, they were presented with some information about the study, after which they completed an online consent form. Participants were then presented with detailed instructions explaining that the survey was run to improve the presentation of financial information. Participants were instructed to make each choice as if they were making such decision in real life. Participants were reminded that there were no right or wrong answers and that they should have made the choice that most suited their personal preference. Participants made each choice between the safe option and the risky prospect by clicking on the corresponding radio button. As soon as a choice was made, a new choice was presented with each choice being presented in isolation on the screen. The order of the above described tasks was the same for all participants, and it adhered to the following sequence: (1) demographics (gender, age, education level and occupation), (2) pre-test, time 1 PaNAS, (3) the 112 choices in the seven different scenarios, (4) post-test, time 2 PaNAS, (4) the four items to elicit subjective evaluations about the emotional nature of the background picture and finally (5) the seven questions about self-rated experience with each of the seven different financial scenarios. 


\section{Results}

\subsection{Data Check}

Before running the analyses, we excluded participants whose total risk propensity (i.e., the average proportion of risky choice across the seven scenarios) was either smaller than .02 or greater than .98 . This measure was taken in order to exclude from the analyses those participants who simply went through the choices and by default almost always chose either the safe option or the risky prospect. A total of 15 participants (i.e., 5.8\%) were excluded as a result of this criterion, reducing the total sample size for the analysis to $\mathrm{N}=245$. We then tested the internal reliability of the choice responses; the analysis returned a Cronbach's $\alpha=.64$, indicating a satisfactory internal consistency, given also the low number of items (i.e., the seven financial scenarios). This result ruled out the possibility that participants responded randomly across the 16 gambles in each scenario due to lack of interest, attention or incentives.

\subsection{Descriptive and Correlational Analyses}

Overall, participants exhibited the highest propensity for risk in the salary domain ( $M=$ $.47, S D=.30)$, and the lowest for insurance $(M=.39, S D=.29)$. Table 1 below shows the proportion of risky choices made depending on financial scenario and valence priming condition. A number of financial scenarios seemed relatively unaffected by the valence priming condition (gamble to win, investment and salary), whilst some variation can be noticed in other domains (gamble to lose, insurance, mortgage and pension). Arousal did not seem to impact on risk taking preferences, as the risk taken across the seven scenarios was comparable between low $(M=.46$, $S D=.20)$ and high arousal $(M=.41, S D=.21)$ priming conditions- even though it was somewhat lower in the latter. 
Table 1. Mean risk taken (and SD) as a function of financial scenario and valence priming.

\begin{tabular}{cccc}
\hline \multirow{2}{*}{ Financial Scenario } & \multicolumn{3}{c}{ Valence priming } \\
\cline { 2 - 4 } & Negative & Neutral & Positive \\
\hline Gamble to win & .46 & .45 & .46 \\
& $(.30)$ & $(.28)$ & $(.29)$ \\
Gamble to lose & .37 & .45 & .41 \\
& $(.33)$ & $(.30)$ & $(.31)$ \\
Insurance & .33 & .44 & .39 \\
& $(.30)$ & $(.30)$ & $(.29)$ \\
Investment & .45 & .46 & .46 \\
& $(.30)$ & $(.29)$ & $(.30)$ \\
Mortgage & .45 & .39 & .44 \\
& $(.31)$ & $(.29)$ & $(.32)$ \\
Pension & .46 & .42 & .42 \\
& $(.29)$ & $(.28)$ & $(.32)$ \\
Salary & .48 & .45 & .48 \\
& $(.30)$ & $(.30)$ & $(.31)$ \\
\hline
\end{tabular}

Table 2 below shows the correlations between the risk taken in the seven different financial scenarios. Overall, the associations were rather strong, indicating somewhat relatively stable risk preferences. However, by further inspecting the association pattern it can be noticed that gamble to lose $(.15<r s<.24)$ and insurance $(.23<r s<.36)$ were somewhat more weakly associated with the other financial domains, which more strongly correlated with each other (.43 $<r \mathrm{~s}<.64)$. 
Table 2. Pearson's $r$ correlation coefficients between the proportions of risky choices in each financial domain.

\begin{tabular}{lllllll}
\hline & 1 & 2 & 3 & 4 & 5 & 6 \\
\hline 1. Gamble to win & & & & & & \\
2. Gamble to lose & .20 & & & & & \\
3. Insurance & .36 & .23 & & & & \\
4. Investment & .63 & .20 & .32 & & & \\
5. Mortgage & .61 & .16 & .35 & .59 & & \\
6. Pension & .59 & .18 & .29 & .60 & .63 & \\
7. Salary & .44 & .19 & .24 & .55 & .46 & .52 \\
\hline
\end{tabular}

Note. All $p$ s $<.01$ apart from the correlation between mortgage and gamble to lose $(p<.05)$

\subsection{Exploratory Factor Analysis}

A principal component analysis (PCA) with oblique rotation (Promax, $\kappa=4$; in order to allow for the possible correlations between the extracted factors) was run and revealed a twofactor solutions that explained a total of $64.5 \%$ of the variance (see Table 3 below). 
Table 3. Two-factor PCA solution: communalities, loadings of variables on factors and percent of variance explained.

\begin{tabular}{cccc}
\hline Scenarios & Communalities & $\begin{array}{c}\text { Factor 1 } \\
\text { ("Positive") }\end{array}$ & $\begin{array}{c}\text { Factor 2 } \\
\text { ("Negative") }\end{array}$ \\
\hline Pension & .69 & .85 & \\
Investment & .70 & .84 & \\
Mortgage & .68 & .83 & \\
Gain & .66 & .79 & \\
Salary & .51 & .71 & .93 \\
Loss & .81 & & .56 \\
Insurance & .47 & & 14.0 \\
& & & 50.5 \\
\end{tabular}

Notes. Loadings of variables on factors are obtained from the pattern matrix to ease interpretation (as shared variance is omitted). Variables are ordered and grouped by size of loadings to facilitate interpretation. For the same reason, loadings under .45 (i.e., $20 \%$ of variance) are omitted.

The interpretation of the solution is relatively straightforward and it supports previous evidence that financial situations were perceived differently by participants (Vlaev et al. 2010). More specifically, gamble to win, pension, investment, mortgage and salary (the 'positive' factor) represent 'positive' financial scenarios where 'positive' utility (e.g., the chance of winning money or earning a salary) is most accessible in mind. On the other hand, for gamble to lose and insurance (the 'negative' factor) the framing is negative possibly due to their 'disutility' connotation.

\subsection{The Effect of Priming on Affect}

A 2 (arousal priming: low vs. high) $\times 3$ (valence priming: negative, neutral, and positive) between-subjects MANOVA on post-experimental negative and positive affect was run. This analysis revealed that the main effect of priming arousal was not significant, $F(2,238)<1$, 
indicating that the arousal priming manipulation did not impact on post-experimental positive and negative affect.

On the other hand, the MANOVA showed that the main effect of priming valence was significant, $F(4,478)=3.3, p=.011$. Follow-up ANOVAs revealed that such effect was significant for negative affect, $F(2,239)=5.2, p=.006$, but not for positive affect, $F(2,239)=$ $2.4, p=.091$. This analysis revealed that participants in the negative priming condition reported significantly higher negative affect than participants in the neutral and positive priming condition (both $p$ s <.008; see Figure 1, panel A).
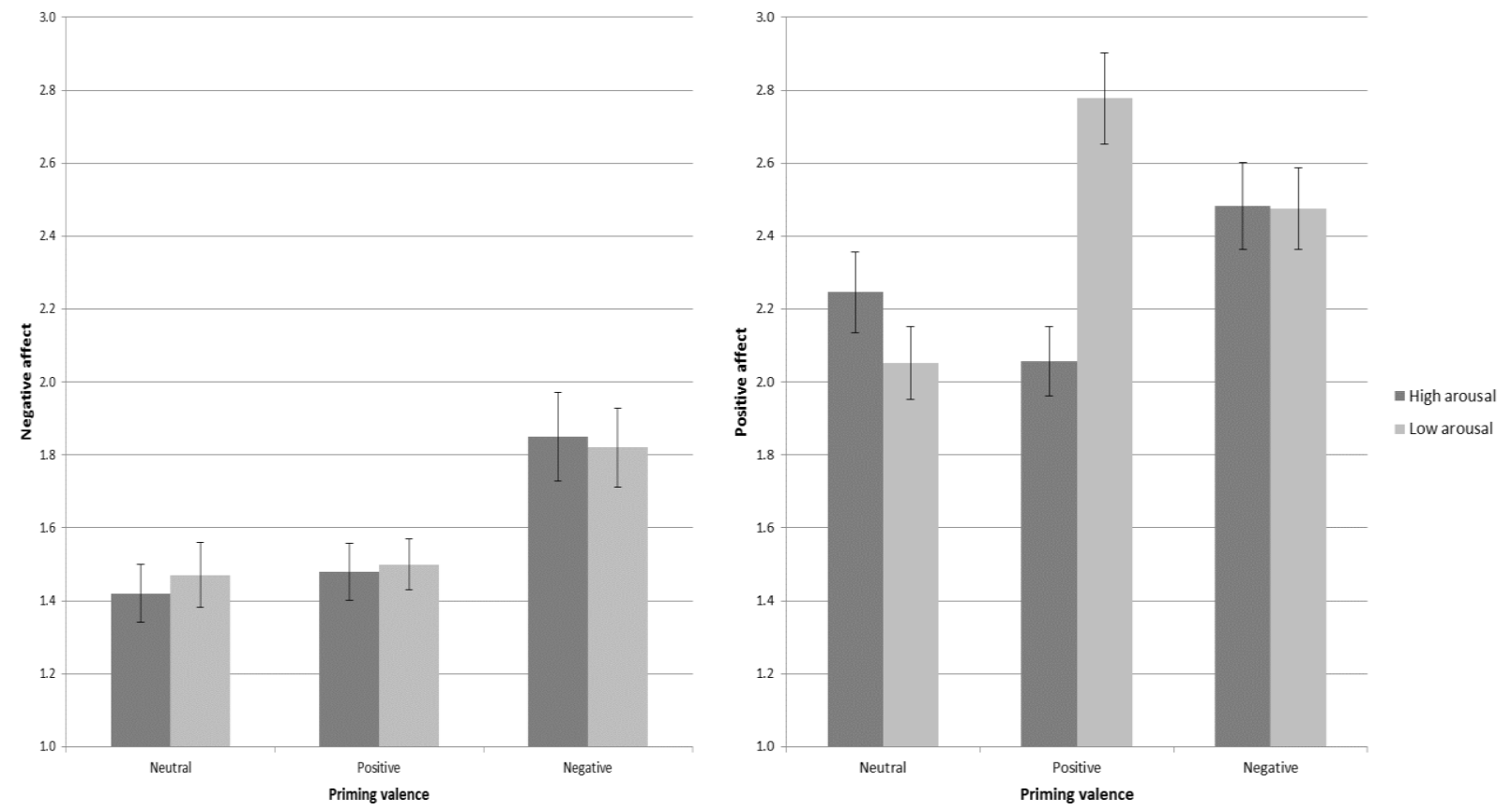

Figure 1. Post-experimental negative (panel A, let) and positive affect (panel B, right) as a function of valence (neutral, negative and positive) and arousal priming (low and high). Error bars represent SEM.

Finally, the MANOVA revealed that the interaction between valence and arousal was significant, $F(4,478)=2.8, p=.025$. Follow-up ANOVAs revealed that such effect was significant for positive affect, $F(2,239)=5.0, p=.008$, but not for negative affect, $F(2,239)<$ 1. This analysis revealed that the effect of priming valence on positive affect depended on the arousal priming level: Whilst there was no significant difference in positive affect for 
participants in the negative and neutral valence condition depending on arousal level (both $p s>$ .357), participants in the positive valence and low arousal priming condition reported significantly higher levels of affect than participants in the positive valence and high arousal priming condition ( $p=.002$; see Figure 1, panel B).

\subsection{The Effects of Priming on Risky Choices}

In order to investigate the effects of priming on risk seeking behavior, a 2 (arousal: low vs. high) $\times 3$ (valence: neutral, negative and positive) $\times 2$ (financial factor: positive vs. negative) mixed ANOVA was run on the proportion of risk taken, with financial factor as the withinsubjects independent variable. Of all the effects, only the main effect of financial factor, $F(1$, $239)=8.4, p=.004$, and the interaction between financial factor and priming valence, $F(2,239)$ $=4.3, p=.015$, were significant (all other $p \mathrm{~s}>.15$ ). The former significant effect showed that, in line with Vlaev et al. (2010), participants made fewer risky choices in the negative financial scenarios $(M=.40, S D=.24)$ compared to the positive scenarios $(M=.45, S D=.23)$. The interaction between financial factor and priming valence is graphed in Figure 2 below, which represents the mean proportion risk taken in each of the two financial factors depending on the valence of the priming condition.

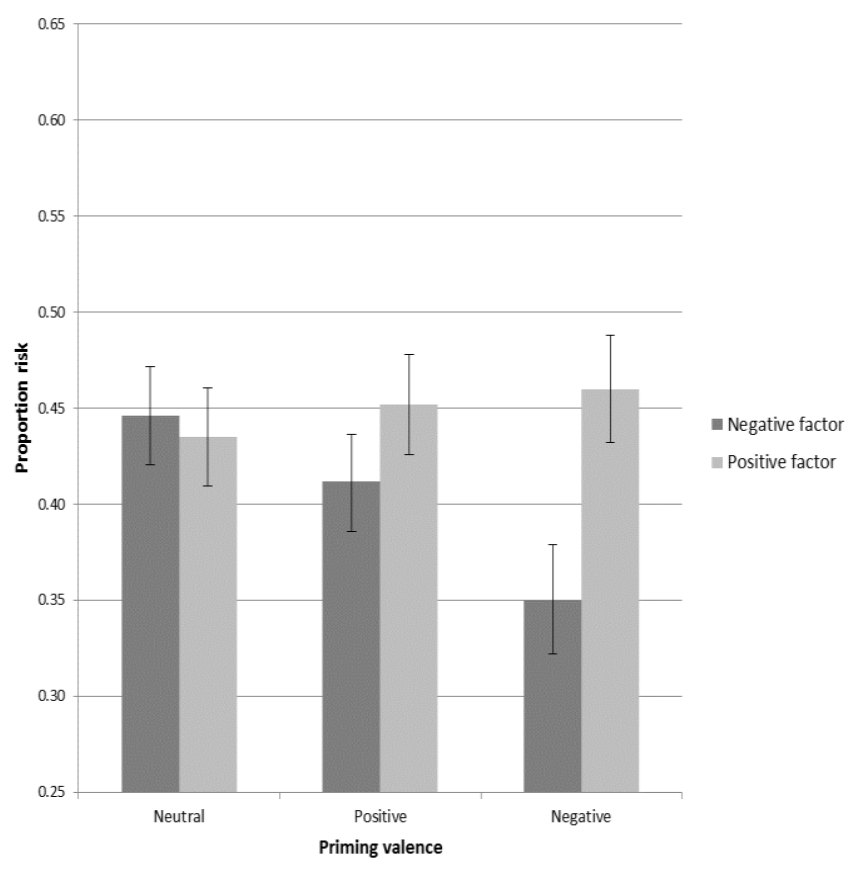

Figure 2. Proportion risk taken as a function of financial factor (positive vs. negative) and priming valence (neutral, negative and positive). Error bars represent SEM. 
In order to break down the significant interaction, three paired samples $t$-tests were run. Risk taken was the same across the two different financial factors when the background picture was either neutral, $t(77)=0.4, p=.694$, or positive, $t(89)=1.4, p=.171$. However, when the background picture was negative in valence, significantly fewer risky choices were made in the negative financial scenarios $(M=.35, S D=.25)$ compared to the positive scenarios $(M=.46, S D$ $=.24), t(76)=3.9, p<.001 .^{1}$

\section{Discussion}

The present study, to our knowledge, offers the first systematic investigation of the effects of valence priming on risk preferences in different financial domains. The results of this experiment show that the effects of valence priming are sensitive to the financial scenario under consideration. First, the arousal component of the priming manipulation did not affect risk taking, and this result addresses the debate in the literature about the multi-component nature of affect priming by suggesting that in the domain of risk preferences, valence-rather than arousal—influences people's decision-making regarding risk.

Second, affect influenced decision-making when there was an 'alignment' between the valence of the priming manipulation and that of the financial context under consideration. Priming did not influence risk taking regardless of the scenario at hand, but rather 'negative' financial scenarios (gamble to lose and insurance) — where the focus might be more closely directed to the potential 'loss' inherent to the financial product - were impacted by negative priming. More specifically, risk aversive choices were more frequent due to negative priming only when participants were making decisions about 'negative' financial products and not for positive ones.

These findings are line with the arguments proposed by Seo et al. (2010) who argued that mood congruence is a more likely effect to observe compared to either mood repair or maintenance when the current decision frame (gain vs. loss) matches the valence of the decision makers' currents feelings (here, the priming manipulation). Indeed, this is what we observed, although this pattern was limited to the negative financial domains. Seo et al.'s (2010) explanation hinges on mood congruency to enhance the salience of future losses; in their study, individuals who experienced a loss and unpleasant feelings became less risk seeking, which

\footnotetext{
${ }^{1}$ Secondary analyses on the (1) associations between subjective ratings of the priming pictures and affect, and (2) role of experience with the financial products are presented in Appendix D.
} 
points towards the increased subjective probability of future losses. Similar results were observed by Au et al. (2003) who examined foreign exchange trading and observed that traders avoided risky decisions more frequently when experiencing unpleasant feelings - in line with the mood congruence effect.

On the surface, these results are at odd with strands of previous literature. As previously mentioned, mood repair and mood maintenance effects are observed when people take more risk to change the current unpleasant feelings and when people avoid choices that could change the current positive state - neither was observed in the present experiment. Moreover, framing effect suggests that people tend to be more risk-seeking when facing negatively framed choices or after experiencing losses (e.g., Kühberger 1998). However, in comparison to previous research, the present experiment systematically investigated the effects of valence priming in relation to different financial products, thus offering a more finely grained investigation on priming and risk taking within the financial domain. Indeed, we show that the specific financial scenario under consideration is differentially impacted by affect. This outcome is generally in line with findings from the research by Isen and her colleagues (e.g., Nygren et al. 1996), who propose that situational factors can moderate the role of affect on risky decision-making. Here, we suggest that the financial product per se can determine how people 'interpret' the decision-making context. More specifically, when people are facing an insurance-related choice, the 'loss' component of the situation becomes more salient and thus people tend to display risk-averse behavior to a greater extent. Moreover, negative affect-here operationalized through a simple priming manipulation — can make the 'loss' component even more salient, thus leading to even more risk-averse behavior.

These findings address the debate on the nature of the dynamics that relate affect to cognitive processing and risky decision-making. As in Seo et al. (2010), these findings suggest that the influence of affect on risk taking was not direct, but it rather interacted with cognitive processes underpinning contextual effects (here as the financial products) in decision-making. Research has already suggested that feelings moderate risk-taking behavior, whereby decisionmakers experiencing unpleasant feelings may avoid risky choices (e.g., Au et al. 2003; Peters and Slovic 2000). Here, we propose that the financial situation framed as an insurance stresses the 'negative' or 'loss-related' element of the choice scenario (here, the potential loss due to a decrease in value of shares) and thus it may be more likely to be associated with unpleasant 
feelings. Relatedly, affect experienced at the moment of decision-making has been proposed to influence cognitive processing in different ways. For example, affect can influence how people estimate the subjective probability of the events to occur (e.g., Rottenstreich and Hsee 2001) or it can impact utility judgments associated with possible outcomes (e.g., Finucane et al. 2000). Within the former mechanism, the mood congruency hypothesis proposes for instance that negative affect is associated with an increase of the estimate of the probability of a negative outcome to occur (e.g., Rottenstreich and Hsee 2001), possibly due to the heightened encoding and retrieving of negative memories, which can directly influence people's choices (e.g., Kusev et al. 2009, 2012).

However, the above findings and considerations need to be considered with caution due to the limitations marring the present study. The priming manipulation was asymmetrical in its effectiveness. On the one hand, negatively valenced background pictures were associated with higher post-experimental negative affect (as measured through PaNAS, Watson et al. 1988), regardless of arousal priming. On the other, higher positive affect was reported only by participants who were presented with the binary choices on a background depicting a positively valenced image associated with low arousal ratings (Lang et al. 1999). This overall asymmetry between negative and positive priming can be readily explained as negative events and stimuli usually evoke greater immediate responses than positive ones (e.g., Taylor 1991). However, according to this explanation, higher affect should be elicited by highly arousal stimuli—rather than by a stimulus associated with low arousal rating as observed in the present study for the positive valence priming manipulation. It is important to note that in the present study normative ratings were utilized when selecting the background priming picture, and thus idiosyncratic effects cannot be ruled out as no pilot study was conducted. Furthermore, future studies could more systematically investigate the different emotions that are usually categorized under the umbrella of negative or positive affect. For instance, different types of negative emotions and affect such as fear, anger and disgust can impact differently on risk-related decision-making (e.g., Lerner and Keltner 2000).

Nonetheless, the results have the potential to offer practical implications. First, they show that people are sensitive to the financial domain in which the choices are framed (see also Vlaev et al. 2010). The relatively different risky choice patterns for the two financial factors (positive vs. negative) suggest that people's financial decision-making is not fully driven by a 'stable risk 
personality', but rather that it is sensitive to the decision context (see also Blais and Weber 2006; Weber, Blais, and Betz 2002). This issue should be considered when financial products are offered to individuals in order to improve the quality of their choice. Service providers should be wary of making simplifying assumptions, such as assuming that a person who makes relatively risk-seeking choices in their investment portfolio is also more likely to avoid displaying riskaverse behavior when facing insurance-related choices. Second, the observed increased risk aversion for 'negative' financial products as a result of negative affect suggests that when people experience unpleasant feelings, they may tend to overestimate the likelihood of negative outcomes occurring, or to anticipate greater dissatisfaction for potential losses. This tendency would lead the decision-maker to be more 'vulnerable' to non-optimal decision-making, especially in relation to insurance-related decisions. In fact, previous research has shown that this may not be the case for all financial products. Au et al. (2003) found that traders in a positive mood overall displayed a worse performance, which was attributed to traders being in a good mood making less accurate decisions. On the other hand, traders in a negative mood made the most accurate decisions but behaved conservatively in their trading. Third, the findings suggest that background images have the potential to influence risky decision-making through their impact on affect. Notwithstanding the above discussed limitations about the priming paradigm, the results suggest that visual information processed during the decision-making task can alter preferences (see also Vohs et al. 2006). For example, background negatively valenced images can increase risk aversion if the financial product that is being considered is insurance. On the other hand, if the financial product under consideration is 'positive' in nature (e.g., it represents an investment or mortgage decision), then the impact of background visual information on risky decision-making can be expected to be negligible.

In conclusion, the present experiment showed that people's decision-making is sensitive to the financial context in which a choice is framed. Moreover, negative affect induces even greater risk aversion for financial products that emphasize the disutility of the choice scenario (e.g., insurance), suggesting that even when the outcome and the probabilities of a choice are known, emotional cues influence risk preferences. 
Conflict of Interest: The authors declare that they have no conflict of interest. 


\section{References}

Aldrovandi, S., Poirier, M., Kusev, P., \& Ayton, P. (2015). Retrospective evaluations of sequences: Testing the predictions of a memory-based analysis. Experimental Psychology, $62,320-334$.

Arkes, H. R., Herren, L. T., \& Isen, A. M. (1988). The role of potential loss in the influence of affect on risk-taking behavior. Organizational Behavior and Human Decision Processes, 42, 181-193.

Au, K., Chan, F., Wang, D., \& Vertinsky, I. (2003). Mood in foreign exchange trading: Cognitive processes and performance. Organizational Behavior and Human Decision Processes, 91, 322-338.

Blais, A. R., \& Weber, E. U. (2006). A domain-specific risk-taking (DOSPERT) scale for adult populations. Judgment and Decision Making, 1, 33-47.

Erb, H. P., Bioy, A., \& Hilton, D. (2002). Choice preferences without inferences: Subconscious priming of risk attitudes. Journal of Behavioral Decision Making, 15, 251-262.

Finucane, M. L., Alhakami, A., Slovic, P., \& Johnson, S. M. (2000). The affect heuristic in judgments of risks and benefits. Journal of Behavioral Decision Making, 13, 1-7.

Forgas, J. P. (1991). Mood effects on partner choice: Role of affect in social decisions. Journal of Personality and Social Psychology, 61, 708-720.

Gilad, D., \& Kliger, D. (2008). Priming the risk attitudes of professionals in financial decision making. Review of Finance, 12, 567-586.

Isen, A. M. (2000). Positive affect and decision making. In M. Lewis \& J. M. Haviland-Jones (Eds.), Handbook of emotions (pp. 417-435). New York: Guilford Press.

Isen, A. M., Nygren, T. E., \& Ashby, F. G. (1988). Influence of positive affect on the subjective utility of gains and losses: It is just not worth the risk. Journal of Personality and Social Psychology, 55, 710-717.

Johansson, P., Hall, L., Sikström, S., \& Olsson, A. (2005). Failure to detect mismatches between intention and outcome in a simple decision task. Science, 310, 116-119.

Kahn, B. E., \& Isen, A. M. (1993). The influence of positive affect on variety seeking among safe, enjoyable products. Journal of Consumer Research, 20, 257-270.

Kühberger, A. (1998). The influence of framing on risky decisions: A meta-analysis.

Organizational Behavior and Human Decision Processes, 75, 23-55. 
Kusev, P., van Schaik, P., Ayton, P., Dent, J., \& Chater, N. (2009). Exaggerated risk: Prospect theory and probability weighting in risky choice. Journal of Experimental Psychology: Learning, Memory, and Cognition, 35, 1487-1505.

Kusev, P., \& Van Schaik, P. (2011). Preferences under risk: content-dependent behavior and psychological processing. Frontiers in psychology, 2, 269.

Kusev, P., van Schaik, P., \& Aldrovandi, S. (2012). Preferences induced by accessibility: Evidence from priming. Journal of Neuroscience, Psychology, and Economics, 5, 250-258.

Kusev, P., Purser, H., Heilman, R., Cooke, A. J., Van Schaik, P., Baranova, V., ... \& Ayton, P. (2017). Understanding risky behavior: the influence of cognitive, emotional and hormonal factors on decision-making under risk. Frontiers in psychology, 8, 102.

Kusev, P., van Schaik, P., Martin, R., Hall, L., \& Johansson, P. (2019). Preference reversals during risk elicitation. Journal of Experimental Psychology: General. Advance online publication. http://dx.doi.org/10.1037/xge0000655

Lang, P. J., Bradley, M. M., \& Cuthbert, B. N. (1999). The international affective pictures system (IAPS). Technical manual and affective ratings. Gainsville, FL: University of Florida.

Lerner, J. S., \& Keltner, D. (2000). Fear, anger, and risk. Journal of Personality and Social Psychology, 81, 146-159.

Loewenstein, G. F., Weber, E. U., Hsee, C. K., \& Welch, E. S. (2001). Risk as feelings. Psychological Bulletin, 127, 267-286.

Mano, H. (1994). Risk-taking, framing effects, and affect. Organizational Behavior and Human Decision Processes, 57, 38-58.

Meyer, J. D., Gaschke, Y. N., Braverman, D. L., \& Evans, T. W. (1992). Mood-congruent judgment is a general effect. Journal of Personality and Social Psychology, 63, 119-132.

Moore, S., \& Chater, N. (2003). The influence of affect on risky behavior: From the lab to real world financial behavior. In Alterman, R., \& Kirsh, P. (Eds.), Proceedings of the 23rd Annual Conference of the Cognitive Science Society. Mahwah, NJ: Lawrence Erlbaum

Nygren, T. E., Isen, A. M., Taylor, P. J., \& Dulin, J. (1996). The influence of positive affect on the decision rule in risk situations: Focus on outcome (and avoidance of loss) rather than probability. Organizational Behavior and Human Decision Processes, 66, 59-72.

Peters, E., \& Slovic, P. (2000). The spring of action: Affective and analytical information processing in choice. Personality and Social Psychology Bulletin, 26, 1465-1475. 
Rottenstreich, Y., \& Hsee, C. K. (2001). Money, kisses, and electric shocks: On the affective psychology of risk. Psychological Science, 12, 185-190.

Seo, M., Goldfarb, B., \& Barrett, L. F. (2010). Affect and the framing effect within individuals over time: Risk taking in a dynamic investment simulation. Academy of Management Journal, 53, 511-531.

Slovic, P. (1995). The construction of preference. American Psychologist, 50, 364-371.

Taylor, S. E. (1991). Asymmetrical effects of positive and negative events: The mobilizationminimization hypothesis. Psychological Bulletin, 110, 67-85.

Tversky, A., \& Kahneman, D. (1979). Prospect theory: An analysis of decision under risk. Econometrica, 47, 263-291.

Vlaev, I., Chater, N., Stewart, N., \& Brown, G. D. A. (2011). Does the brain calculate value? Trends in Cognitive Sciences, 15, 546-554.

Vlaev, I., Kusev, P., Stewart, N., Aldrovandi, S., \& Chater, N. (2010). Domain effects and financial risk attitudes. Risk Analysis, 30, 1374-1386.

Vohs, K. D., Mead, N. L., \& Goode, M. R. (2006). The psychological consequences of money. Science, 314, 1154-1156.

Van Schaik, P., Kusev, P., \& Juliusson, A. (2011). Human preferences and risky choices. Frontiers in Psychology, 2, 333.

Watson, D., Clark, L. A., \& Tellegen, A. (1988). Development and validation of brief measures of positive and negative affect: The PANAS scales. Journal of Personality and Social Psychology, 54, 1063-1070.

Weber, E. U., Blais, A. R, \& Betz, N. (2002). A domain-specific risk-attitude scale: Measuring risk perceptions and risk behaviors. Journal of Behavioral Decision Making, 15, 263-290.

Zajonc, R. B. (1980). Feeling and thinking: Preferences need no inferences. American Psychologist, 35, 151-175. 


\section{Appendix A}

Final scenarios descriptions

1) Gambles with gain

Imagine choosing between "receiving $£ 30$ for certain" or a "50\% chance of winning $£ 100 "$. Which option would you choose? Here you would have to imagine making choices between playing a gamble to receive an amount of money and taking a smaller amount for sure. Each pair of options is presented as two pie charts. Imagine that a spinner is attached to the center of the pie chart and after the spinner is spun you will receive the money written in the region where the pointer lands. As you can see, the pie charts representing 'Sure Amount' will always give you a certain amount while the pie charts representing 'Gamble' offer either a bigger amount or zero (the two regions of these pie charts represent the probabilities for each amount respectively). Please circle the pie chart you would prefer (the sure amount or the gamble) in each pair. Note that there are no correct answers and your choice is a matter of personal preference, but try to choose which option (sure amount or a gamble) you would prefer if this choice was made for real.

\section{2) Investment}

Imagine that you want to make an investment decision and you are offered a choice between buying either a bond that give you $£ 30$ profit for certain per year or buying a company share that has a $50 \%$ chance of bringing you $£ 100$ profit per year. Which option would you choose? Here you would have to imagine making choices between buying a company share with an uncertain profit and buying a bond offering a smaller profit for sure. Each pair of options (bond versus share) is presented here as two pie charts. Imagine that a spinner is attached to the center of each pie chart and after the spinner is spun you will receive the money written in the region where the pointer lands. As you can see, the pie charts representing 'Bonds' will always give you a certain profit while the pie charts representing 'Shares' offer either a bigger profit or zero (the two regions of these pie charts represent the probabilities for each profit respectively). Please circle the pie chart you would prefer (bond or share) in each pair. Note that there are no correct answers and your choice is a matter of personal preference, but try to choose which option (bond or share) you would prefer if this choice was made for real. 


\section{3) Mortgage}

Here you are offered pairs of houses and you have to choose which house to buy with a mortgage. However, in order to repay for the mortgage, you need to rent part of the house because your salary is not enough. One of the houses in each pair is always in a location within the town, which will give you a stable moderate average profit per week because it is in a road that is desirable, has similar houses and therefore has a stable regular market of interested tenants. The other house could bring you a bigger profit with certain probability because of its specific location that makes it desirable to only a smaller number of tenants who will be prepared to pay over the odds for it (and you can save for future mortgage payments). However, there is also a chance of not making any profit from this house because you might not be able to find tenants for the whole year (and you would have borrow money to repay the mortgage). Each pair of houses is presented as two pie charts. Imagine that a spinner is attached to the center of the pie chart and after the spinner is spun you will receive the profit written in the region where the pointer lands (which is the average weekly profit throughout the year). The pie charts representing 'Guaranteed Profit House' will always give you a certain profit. The pie charts representing 'Variable Profit House' offer either a bigger profit or a zero profit, and the two regions of these pie charts represent the probabilities for each profit respectively. Please circle the pie chart (house) you would prefer in each pair. Note that there are no correct answers and your choice is a matter of personal preference, but try to choose which house you would prefer if this choice was made for real.

\section{4) Salary}

Imagine that you are the only income earner in the family and you are searching for a job and you are offered to choose between two jobs. One job is offering $£ 30$ daily payment for certain while the other job is offering you variable payment scheme with a $50 \%$ chance of receiving salary of $£ 100$ per day because the payment depends on the company’s performance for each day. Which option would you choose? Here you would have to imagine making choices between taking a job that offers you variable daily payment (salary) and taking job offering a smaller payment for sure. Each pair of jobs (payment schemes) is presented as two pie charts. Imagine that a spinner is attached to the center of the pie chart and after the spinner is spun you 
will receive the daily salary written in the region where the pointer lands. As you can see, the pie charts representing 'Fixed Salary (per day)' will always give you a certain amount while the pie

charts representing 'Variable Salary (per day)' offer either a bigger daily salary or zero (the two regions of these pie charts represent the probabilities for each salary respectively). Please circle the pie chart (job) you would prefer (the fixed salary or the variable salary) in each pair. Note that there are no correct answers and your choice is a matter of personal preference, but try to choose which job you would prefer if this choice was made for real.

\section{5) Pension}

Imagine you are saving for a pension (retirement income) and you are offered to choose between two pension investment plans. You can either invest your money safely in bonds with a fixed interest rate, thus offering a smaller pension for sure, or make a riskier stock market investment in company shares which could make you more money (bigger pension) but might also lose your savings if the stock market fails. For example, imagine choosing between receiving pension of $£ 30$ per day for certain or a $50 \%$ chance of receiving $£ 100$ pension per day. Which option would you choose? Here each pair of expected pensions (per day) is presented as two pie charts. Imagine that a spinner is attached to the center of the pie chart and after the spinner is spun you will receive the pension written in the region where the pointer lands. As you can see, the pie charts representing 'Sure Pension (per day)' will always give you a certain pension, while the pie charts representing 'Variable Pension (per day)' offer either a bigger pension or zero (the two regions of these pie charts represent the probabilities for each pension respectively). Please circle the pie chart you would prefer (sure pension or variable pension) in each pair. Note that there are no correct answers and your choice is a matter of personal preference, but try to choose which pension investment plan you would prefer if this choice was made for real.

\section{6) Gambles with loss}

Imagine choosing between "losing $£ 30$ for certain" or a "50\% chance of losing $£ 100 "$ (and hence there is a 50\% chance of not losing anything). Which option would you choose? Here you would have to imagine making choices between playing a gamble that can make you lose an amount of money and losing a smaller amount for sure. Each pair of options is again 
presented as two pie charts. Imagine that a spinner is attached to the center of the pie chart and after the spinner is spun you will lose the money written in the region where the pointer lands. As you can see, the pie charts representing 'Sure Loss' will always make you lose a certain amount while the pie charts representing 'Loss Gamble' can make you lose either a bigger amount or zero (the two regions of these pie charts represent the probabilities for each loss respectively). Please circle the pie chart you would prefer (sure loss or gamble) in each pair. Note that there are no correct answers and your choice is a matter of personal preference, but try to choose which option (sure loss or gamble) you would prefer if this choice was made for real.

\section{7) Insurance}

Imagine that you possess 16 shares of different companies, which are worth now $£ 100$, $£ 200, £ 300$, or $£ 400$ respectively. The value of each of these shares is however in danger of shrinking due to a fall in its popularity. The extent of this loss in value depends on how much the popularity of the share (company) decreases and is measured by the probability of each asset losing its total value (going to zero). This could be determined by the general economic and market conditions or because the particular company underperforms. You have the possibility of insuring yourself against the possible loss in each share's value. If you insure yourself, you will be compensated for any loss in value. To insure yourself you must pay the price of an insurance premium. Each share's probability of losing its value and the corresponding insurance cost are presented here as two pie charts. Thus, one pie chart is the 'Insurance Cost', which is fixed for each share and in the first example below it is $£ 4$. The other pie chart represents the 'Probability of Value Loss' (the probability that the share will lose its value) and in the first example below there is a $20 \%$ chance that the first share worth $£ 100$ will lose its value (the two regions of these pie charts represent the probabilities for each value loss respectively). Imagine that a spinner is attached to the center of the pie chart and after the spinner is spun your share will lose the value written in the region where the pointer lands. Please circle the pie chart you would prefer in each pair (pay insurance or accept the chance). Note that there are no correct answers and your choice is a matter of personal preference, but try to choose which option (pay insurance or accept the chance) you would prefer if this choice was made for real. 


\section{Appendix B}

Presentation of amounts and probabilities in the gamble task

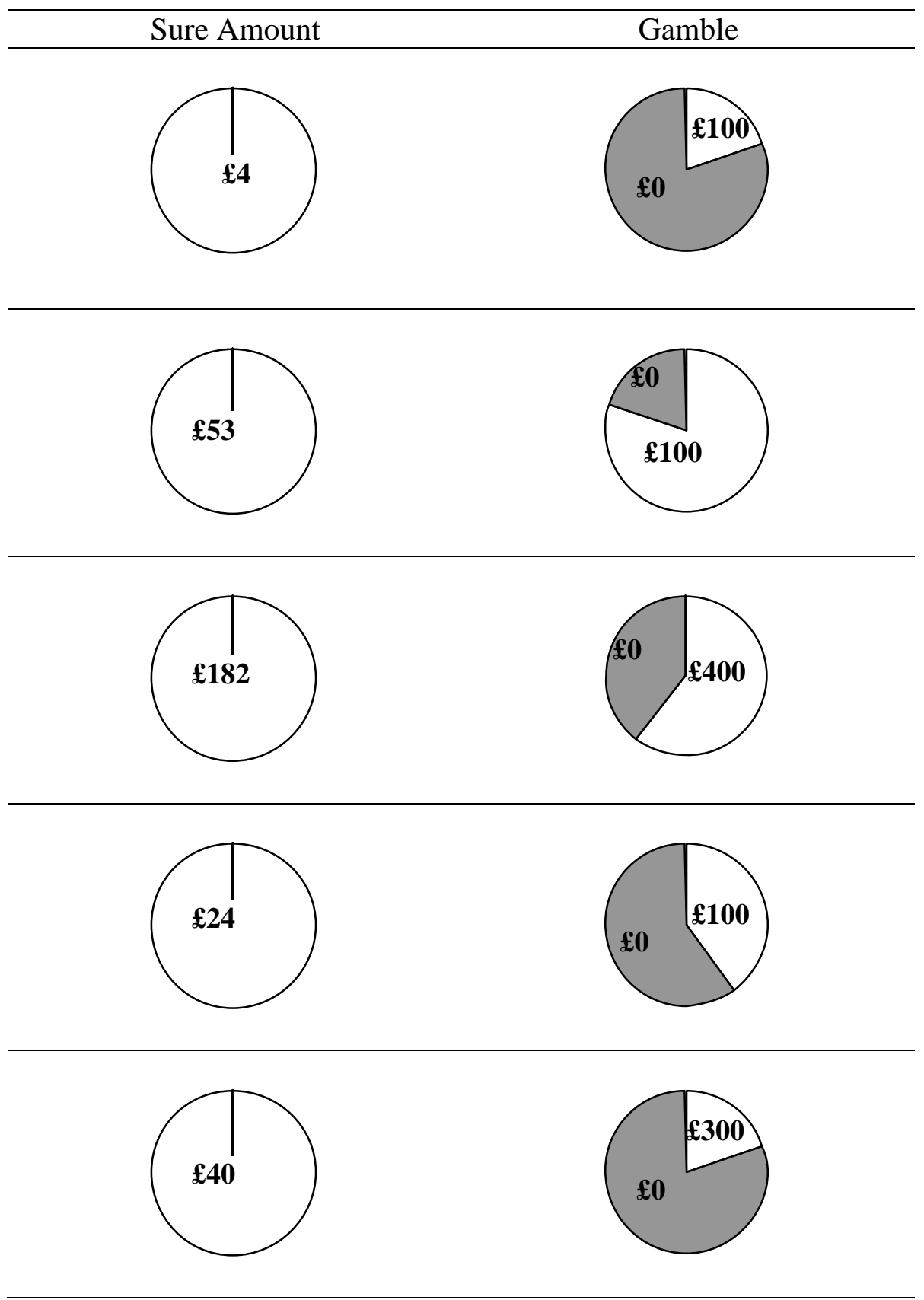




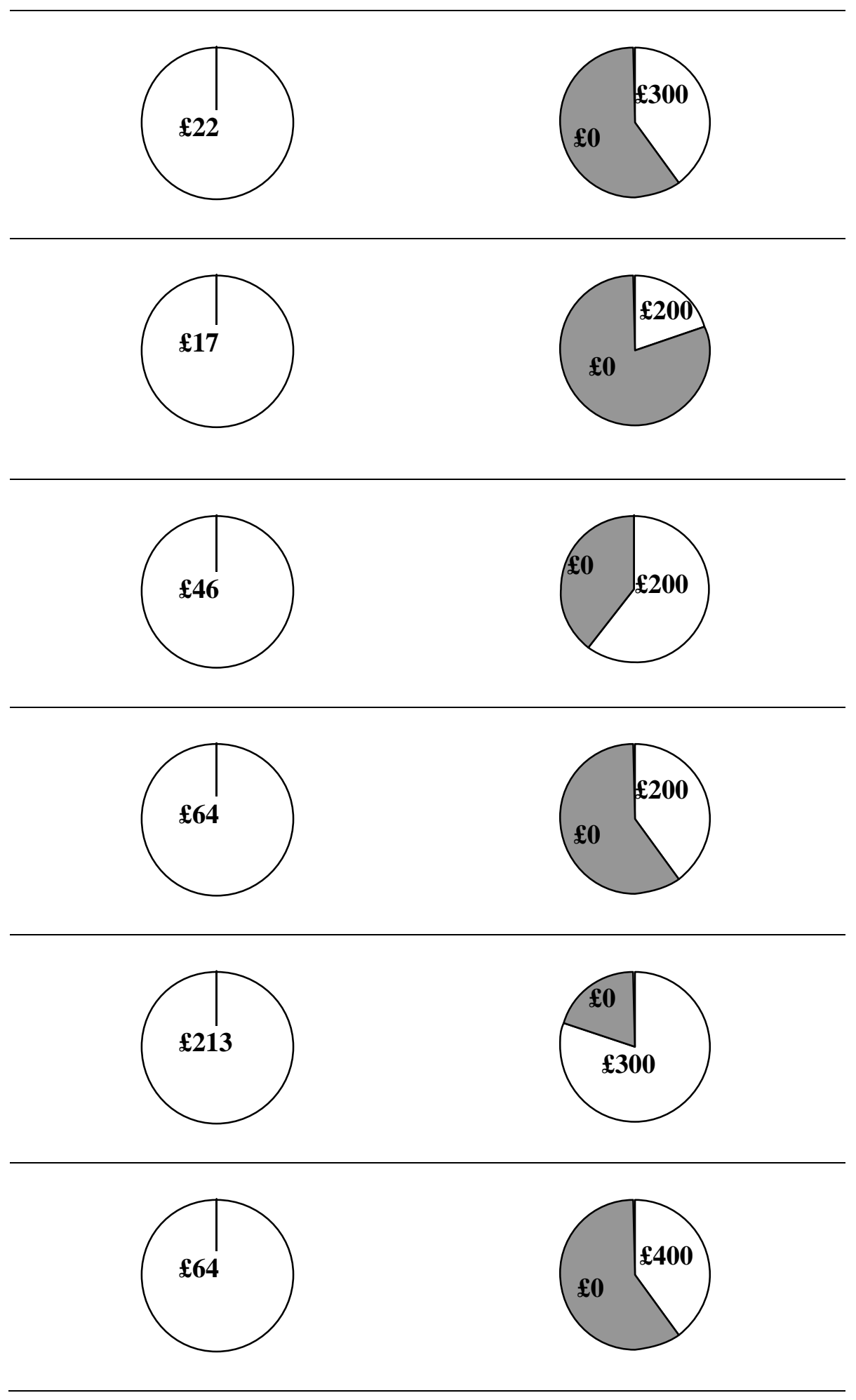




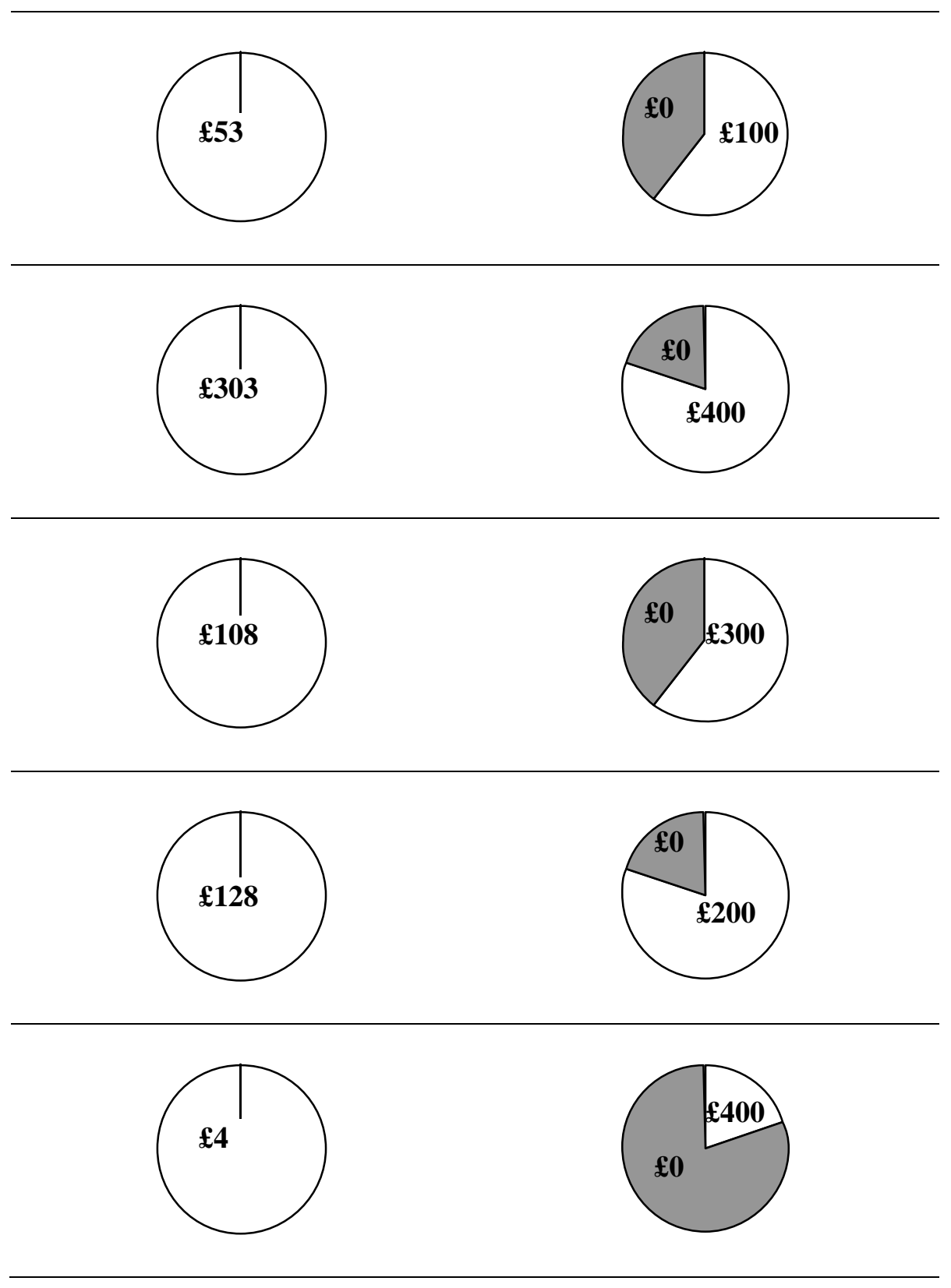




\section{Appendix C}

Priming background pictures
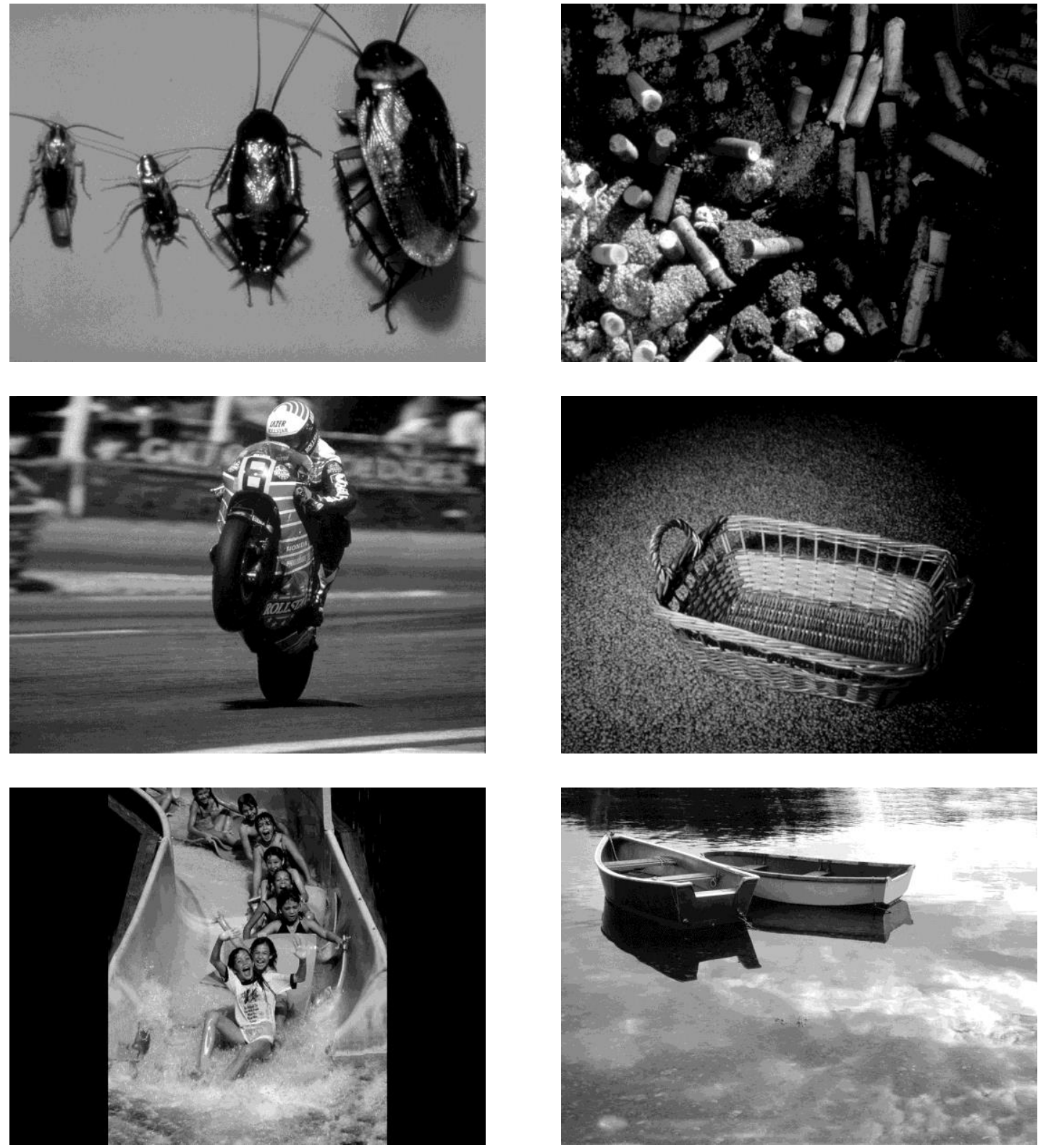

The first row presents the negative pictures (high arousal, left; low arousal, right), the second the neutral pictures and finally the third row the positive pictures. Within brackets we list the picture number as listed in the IAPS database and the average valence and arousal ratings for each picture, respectively: (1) cockroaches (1274; 1.4, 4.7); (2) cigarette (9830; 2.6, 2.5); (3) motorbike $(8250 ; 4.8,4.4)$; (4) basket $(7010 ; 4.4,1.6)$; (5) water slides $(8496 ; 7.6,4.7)$; and (6) boat $(5390 ; 6.0,2.0)$. 


\section{Appendix D}

Additional analyses on the (1) associations between subjective ratings of the priming pictures and affect, and (2) the role of experience with the financial products

We ran correlation analyses to inspect any association between participants' subjective evaluations of the background picture with post-experimental affect measures. Some significant associations were noted, although they were few and weak. For instance, as expected, ratings of the picture as (1) 'negative, unhappy' correlated with negative affect, $r=.16, p=.011$, and (2) 'positive, happy' was significantly associated with positive affect, $r=.23, p<.001$. Finally, positive affect also correlated with ratings of 'excited, aroused', $r=.15 . p=.021$.

The role of experience with the financial products was also explored. We first computed the average experience ratings across the two domains of the negative factor and the five products belonging to the positive factor. We then correlated the experience ratings with the risk taken. The analyses revealed that the experience between the two factors correlated strongly, $r=$ $.69, p<.001$. More importantly, only the experience for the two negative products correlated with the risk taken for the negative factor, $r=.14, p=.031$. All other correlations were nonsignificant, $|r s|<.10$. 